\title{
Activité musculaire des membres inférieurs en course à pied sur le plat
}

\section{Christine HANON,}

Laboratoire de biomécanique et de physiologie, DSS, INSEP

11 avenue du Tremblay 75012 Paris

Tél. : 0141744165

Fax : 0141744535

Courriel : christine.hanon@insep.fr

\section{RÉSUMÉ :}

Cet article a pour objectif principal de décrire l'activité des principaux muscles des membres inférieurs dans la course à pied sur le plat. L'examen de la littérature à ce sujet permet de mettre en avant le fait que les muscles mono-articulaires sont activés une seule fois au cours du cycle de la foulée (vastus lateralis, gluteus maximus). Les muscles bi-articulaires sont activés deux fois : quelle que soit la vitesse pour le biceps femoris et une ou deux fois selon la vitesse pour le rectus femoris et le gastrocnemius. De ce fait, certains muscles sont activés fortement mais brièvement (gastrocnemius, vastus lateralis), d'autres sont activés faiblement mais longtemps (tibialis anterior) et d'autres encore tels que le rectus femoris et le biceps femoris sont activés assez longuement et assez fortement.

L'élévation de la vitesse influe très largement sur ces données en augmentant les niveaux d'activation et en modifiant peu ou prou les patterns d'activation. Les conséquences liées à la fatigue se traduisent par une augmentation du rapport temps de travail / temps de relaxation, et à une augmentation du rapport EMGi / force. Enfin, les conséquences du niveau d'expertise sur les patterns et niveaux d'activité, quoique peu abordées dans la littérature, sont présentées dans leur lien avec le coût énergétique et dans l'évolution de ces patterns avec la fatigue.

KEY WORDS : course, activité musculaire, membres inférieurs, vitesse, fatigue, expertise.

\section{ABSTRACT :}

Muscular activity of the lower limbs during flat running

The primary objective of this article is to describe the main muscle activity of the lower limbs during flat running. The examination of the literature on this subject emphasizes the fact that the mono-articular muscles are only once activated during the stride cycle (vastus lateralis, gluteus maximus). The bi-articular muscles are activated twice: whatever the speed for the biceps femoris and once or twice depending on the speed for the rectus femoris and the gastrocnemius. From this, certain muscles are activated strongly but briefly (gastrocnemius, vastus lateralis), while others are activated weakly but for a long time (tibialis anterior) and others still, such as the rectus femoris and the biceps femoris are activated long and strongly.

Raising the speed has a significant impact on these data by increasing the levels of activation and by modifying more or less the activations patterns. The consequences of fatigue result in an increase in the ratio period of work / period of relaxation, as well as in an increase in the relationship EMGi / Force. Finally, the consequences of the level 
of expertise on patterns and levels of activity, although discussed only infrequently in the literature, are presented in relation to their role with regard to both the energetic cost and the evolution of these patterns with fatigue.

KEY WORDS : running, muscular activity, lower limbs, speed, fatigue, expertise.

\section{INTRODUCTION}

L'objet de cette présentation est de décrire les activités musculaires des principaux muscles des membres inférieurs au cours de la course sur le plat en centrant les informations sur 4 points :

- période d'activité (situer l'activité musculaire dans le cycle de la foulée) ;

- durée d'activité (en valeur absolue soit en msec et/ou en valeur relative, soit en \% de la durée du cycle de la foulée) ;

- le niveau d'activité (exprimé en \% d'un niveau d'activité musculaire maximale) ;

- modes de contraction musculaire mis en évidence au cours des différentes phases de la foulée.

Dans un second temps, l'influence de la vitesse, de la fatigue et de l'expertise sur les 3 premiers points sera examinée. Ces données ont pu être établies par l'examen de la littérature sur ce sujet. Exception faite de l'analyse des modes de contraction musculaire, toutes sont basées sur la technique de recueil des données électromyographiques (EMG).

\section{DESCRIPTION}

\subsection{Différentes phases de la foulée}

Avant de procéder à l'analyse des activités musculaires, il paraît nécessaire de préciser les différentes phases de la foulée, qui permettront de les situer dans le temps (figure 1).

\subsection{Caractéristiques générales des muscles étudiés}

Afin de préciser les différentes phases articulaires de la foulée (flexion plantaire et dorsale de la cheville, flexion et extension du genou, flexion et extension de la hanche), cette présentation est basée sur une synthèse de l'activité des muscles des compartiments antérieurs et postérieurs aussi bien distaux que proximaux. La proportion de fibres de type II relevée dans la littérature (Bouisset \& Maton, 1995 ; Johnson et al., 1973) est précisée ci-dessous :

- le gastrocnemius (Ga) : 49 à $56 \%$

- le tibialis anterior (TA) : 27 à $40 \%$

- le biceps femoris (BF) : $33 \%$

- le vastus lateralis (VL) : $67 \%$

- le rectus femoris (RF): 58 à $70 \%$

- le gluteus maximus (GM) : $48 \%$ 
On peut noter que certains de ces muscles (TA, VL, GM) sont mono-articulaires et sont sollicités dans le cadre d'une seule fonction (ex. : TA est un fléchisseur dorsal de la cheville), d'autres ( $\mathrm{Ga}, \mathrm{BF}, \mathrm{RF}$ ) sont bi-articulaires et sont donc activés dans le cadre de la flexion d'une articulation et de l'extension d'une autre (ex. : BF est activé au cours de la flexion du genou et de l'extension de la hanche).

\subsection{Périodes d'activité des différents muscles}

La figure 2 résume à partir d'enregistrements électromyographiques les caractéristiques temporelles d'activité de différents groupes musculaires des membres inférieurs

au cours d'un cycle de la foulée (pose talon droit à pose talon droit suivante). Ces enregistrements ont été analysés à $15 \mathrm{~km} . \mathrm{h}-1$. II est possible à partir de ces enregistrements d'observer un nombre variable de bouffées d'activité au cours d'un cycle de foulée selon les muscles considérés. En effet, il est repéré :

- 1 bouffée ( $\mathrm{Ga}$, VL, GM qui sont considérés comme des muscles monophasiques)

-2 bouffées (RF, BF qui sont donc des muscles bi-phasiques en course)

-2 à 3 bouffées (TA)

II est à noter (figure 2 et figure 3 ) que tous ces muscles sont activés fin PO3 et début PA, que PO1 est une phase de relaxation musculaire sauf pour TA et RF. Enfin, on peut remarquer que les bouffées EMG enregistrées en phase d'appui commencent toutes entre 70 et 180 millisecondes avant la pose du talon au sol.

\subsection{Rôle, durée et niveau d'activité des muscles étudiés}

Le rôle (R), la durée (DA), le niveau d'activité (NA) ainsi que le mode d'activité (MA) seront précisés et quantifiés pour chaque muscle. La présentation distingue les résultats obtenus pour des vitesses submaximales inférieures à $25 \mathrm{~km} . \mathrm{h}-1$. Les données issues d'études réalisées sur le sprint sont présentées dans le point 2.1. Les durées d'activités sont exprimées ici en \% de la durée totale du cycle de la foulée. Les degrés d'activité sont exprimés relativement à un niveau d'activité considéré comme maximal et enregistré à l'occasion d'un effort isométrique maximal.

La résistance isométrique est généralement proposée, soit par opposition manuelle dont les conditions sont peu précisées dans la littérature, soit sur des ergomètres spécifiques (Hanon et al., sous presse). Le degré d'activité sera alors exprimé en \% FVM sous-entendu en \% du niveau d'activité recueilli en situation isométrique maximale.

Le mode d'activation est, quant à lui, déterminé par analyse cinématographique à image rapide (Mann et al., 1986) associée éventuellement à un passage sur plateforme de force (Méro \& Komi, 1987) ou par électrogoniomètre (McClay et al., 1990).

\section{Gastrocnemius}

$\mathbf{R}$ : Ga agit sur la flexion du genou et sur la flexion plantaire (appelées parfois extension de la cheville). Nummela et al. (1992) soulignent le rôle majeur joué par ce muscle au cours de la propulsion dans la course où il est actif dès la fin de la phase d'oscillation (PO3). Associé à TA, il a pour rôle de stabiliser le pied dans une position adaptée à la prise d'appui (McClay et al., 1990). II intervient aussi pour décélérer le tibia dans son mouvement vers l'avant. 
DA : D'après Reber et al. (1993), Ga est activé à plus de $25 \%$ de FVM, pendant 30 à $35 \%$ de la durée du cycle de la foulée. Ceci est confirmé par Mann et al. (1986). Sur tapis roulant à $21 \mathrm{~km} . \mathrm{h}-1$, cette durée correspond en moyenne à $233+48 \mathrm{msec}$ et à 38 $\%$ du cycle de la foulée (Hanon et al., sous presse).

NA : À $21 \mathrm{~km} . \mathrm{h}-1$, il correspond à $56 \%$ de FVM (Hanon et al., sous presse)

MA : Avant la pose du pied (PO3), Ga intervient sur le mode isométrique pour stabiliser le pied puis sur le mode excentrique pour décélérer le mouvement vers l'avant du pied. Une contraction concentrique de $\mathrm{Ga}$ n'est observée en fin de phase d'appui qu'aux vitesses les plus élevées (Elliot \& Blanksby, 1979).

\section{Tibialis anterior}

$\mathbf{R}$ : Sa fonction est de relever le pied et de participer à la supination et adduction de la cheville. Il est actif une grande partie du cycle de la foulée.

DA : Il semble selon Reber et al. (1993) que le pattern du TA soit particulier : en effet, ce muscle serait actif pendant $85 \%$. Hanon et al. (sous presse) note même qu'à 21 km.h-1, ce muscle est activé jusqu'à $90 \%$ du cycle de la foulée soit pendant plus de 400 msec. Pour Reber et al. (1993), les autres muscles responsables du contrôle de la cheville ne seraient actifs que pendant $50 \%$ du cycle de la foulée.

Un temps de silence a été observé brièvement après la pose du pied (Nilsson et al., 1985) et au cours de la seconde moitié de l'appui (Mann \& Hagy, 1980).

NA : Le pic d'activité est mesuré au poser du talon. À partir de vitesses supérieures au jogging (et non précisées par les auteurs), le TA n'est jamais activé à moins de $10 \%$ de son activité en situation de FVM. Aux vitesses supérieures au jogging, le TA semble sollicité entre 20 et $50 \%$ de FVM, avec des pics à $60 \%$ aux vitesses les plus rapides (Reber et al., 1993) ce que confirme Hanon (Hanon et al., sous presse) avec une valeur de $42 \%$ à $21 \mathrm{~km}$.h-1. La moyenne du niveau de l'activité à des allures définies comme des allures d'entraînement est de 30 \% de FVM (Reber et al., 1993).

MA : Au moment du poser du talon, TA est activé sur le mode excentrique pendant que l'avant-pied descend vers le sol (selon Elliot \& Blanksby, 1979). Quand le pied est à plat sur le sol, TA semble activé sur le mode concentrique pour participer à amener la jambe vers l'avant (McClay et al, 1990). Pendant les phases d'oscillation, l'activité de TA semble réalisée sur le mode excentrique puis, pour certains sujets, sur le mode concentrique (Simonsen et al., 1985).

\section{Biceps Femoris}

$\mathbf{R}$ : La longue portion de ce muscle est classiquement définie comme un extenseur de la hanche et un fléchisseur du genou par Simonsen et al. (1985), Méro \& Komi (1987), Bober \& Mularez (1990), McLay et al. (1987). Une fois le pied posé au sol et le centre de gravité placé au-dessus du genou, les ischios-jambiers étendent la hanche, tirant en même temps la cuisse vers l'arrière (Steindler, 1964). À la pose du pied, les mises en jeu associées de BF et de GM ont pour conséquence de minimiser la composante horizontale freinatrice de la réaction du sol. Dans l'exemple proposé figure 4, l'activité de BF au cours de la phase d'appui semble très limitée.

Au moment où le pied quitte le sol, la contraction des ischios-jambiers provoque la flexion du genou (PO2). Le rôle de BF sera ensuite de contrôler la flexion de la hanche (Montgomery, 1994), alors que le chef court de BF contrôlera l'extension du genou.

DA : L'activité du BF au cours du cycle de la foulée est, selon Mann et al. (1986), deux fois plus longue que celle du quadriceps. Elle correspond sur l'ensemble des deux bouffées à $75 \%$ du cycle de la foulée soit plus de 400 msec à 21 km.h-1 (Hanon et al., sous presse). 
NA : À $15 \mathrm{~km} . \mathrm{h}-1$, la longue portion du BF est, en effet, activée à plus de $25 \%$ de FVM pendant environ $60 \%$ du cycle de la foulée. La portion courte étant activée également à environ $50 \%$ de FVM.

MA : Les données concernant le mode de contraction pendant l'appui diffèrent selon les auteurs et surtout selon la vitesse. Pour Mann \& Haggy (1980), elle serait isométrique en phase d'appui pour maintenir une longueur constante pendant l'extension du genou et de la hanche. Au début de la phase d'oscillation (PO1), il semble que la flexion du genou soit le résultat de la rapide accélération vers l'avant de la cuisse et soit donc réalisée sans participation active du BF. Pendant PO2, BF est activé sur le mode excentrique afin de décélérer la flexion de la hanche, puis de contrôler l'extension du genou. Ensuite (PO3), BF se contracte sur le mode concentrique pour étendre la hanche et fléchir le genou.

\section{Vastus lateralis}

$\mathbf{R}$ : Les fonctions principales sont l'extension du genou (fin PO3) et le contrôle de la flexion du genou (PA). Selon Montgomery et al. (1994), VL joue un rôle important dans la stabilisation du genou pendant l'appui. Pour Mann et al. (1986), l'extension du genou dans la deuxième partie de l'appui est le résultat du mouvement du corps vers l'avant, sur tibia et pied fixés. La fin de l'activité des vastii serait contemporaine de la fin d'activité du GM (PA).

DA : La durée d'activité de VL est estimée sur tapis roulant à $152 \mathrm{msec}$ soit $30 \%$ du cycle de la foulée (à $21 \mathrm{~km} . \mathrm{h}-1$ ) (Hanon et al., sous presse) ce qui semble cohérent par rapport aux données de Montgomery et al., 1994 : $25 \%$ de la durée du cycle de la foulée à $15 \mathrm{~km} \cdot \mathrm{h}-1$.

NA : Au cours de l'appui, VL est activé au maximum jusqu'à $78 \%$ FVM et en moyenne à $53 \%$ de FVM (Montgomery et al., 1994). Pour Hanon et al., sous presse), les valeurs recueillies sur tapis roulant sont de $60 \%$ de FVM à $21 \mathrm{~km}$.h-1.

MA : Selon Nilsson et al. (1985) et Mann (1986), VL se contracte sur le mode excentrique au début de l'appui alors que la flexion du genou continue pour passer vraisemblablement à un mode de contraction isométrique puis concentrique. En phase d'oscillation, à vitesse élevée, VL se contracte sur le mode concentrique pour étendre le genou au cours de la descente du pied.

\section{Rectus Femoris}

R : Selon Kapandji (1965), RF ne représente que le cinquième de la force totale du quadriceps, mais, bi-articulaire, il agit à la fois comme un fléchisseur de la hanche et comme un extenseur du genou. Son action sur la hanche dépend du degré de flexion du genou et inversement son efficacité comme extenseur du genou dépend de la position de la hanche. Pendant l'appui, RF participe comme les vastii à la stabilisation du genou. II intervient ensuite dans les mouvements associant l'extension du genou et la flexion de la hanche (comme dans l'avancée du membre oscillant) (PO1). Son rôle essentiel semblerait toutefois celui de fléchisseur de la hanche (Méro \& Komi 1987).

Aussi bien à vitesses basses qu'à vitesses élevées, les auteurs tels que Elliott \& Blanksby (1979) et Montgomery et al. (1994) trouvent toujours deux périodes d'activité : une première en phase d'appui et une seconde en phase d'oscillation du pied controlatéral. Ceci semble contredit par Hanon et al. (sous presse) et Wank (1998) qui ne détectent qu'une bouffée aux vitesses les plus basses mais ces résultats sont acquis sur tapis roulant avec des sujets plus experts.

DA : À $21 \mathrm{~km} . \mathrm{h}-1, \mathrm{RF}$ est activé durant $270 \mathrm{msec}$ (réparties en 2 fois) soit $56 \%$ du cycle de la foulée (Hanon et al., sous presse). 
NA : À $15 \mathrm{~km} . \mathrm{h}-1, \mathrm{RF}$ est activé pendant seulement $20 \%$ du cycle de la foulée à plus de $25 \%$ de FVM. II semble être activé à moins de $10 \%$ de FVM, pendant $70 \%$ du cycle de la foulée (Montgomery et al., 1994). Sur tapis roulant à $21 \mathrm{~km} . \mathrm{h}-1$, RF est activé en moyenne à $36 \%$ de FVM, la bouffée enregistrée au cours de l'appui correspondant à l'activité la plus intense.

MA : Selon Nilsson et al., 1985, RF est contracté sur le mode excentrique au début de l'appui alors que le genou se fléchit et que la hanche s'étend. Une phase de sollicitation du muscle sur le mode isométrique et concentrique est ensuite observée. La phase d'oscillation (PO2) sollicite RF sur le mode concentrique ce qui aboutira à la flexion de la hanche. Ces informations peuvent être assez largement nuancées pour ce qui concerne la course à grande vitesse (cf. point 2.1).

\section{Gluteus maximus}

$\mathbf{R}$ : GM contribue avec les ichios jambiers à minimiser la perte de vitesse horizontale au moment du contact au sol (fin PO3 et PA) (Simonsen et al., 1985). En effet, pour Waser (1985), Lemaire \& Robertson (1987) et Ae et al. (1992), GM apparaît comme le principal muscle responsable de l'accélération vers l'avant. Wieman \& Tidow (1995) le qualifient également de muscle antigravitaire.

Néanmoins, ces mêmes auteurs soulignent qu'outre le fait d'être un extenseur de la hanche, GM est également un abducteur ce qui nécessite une intervention compensatrice pour soutenir et neutraliser GM dans son effet d'abduction. L'adductor magnus (AM) semble jouer un rôle fondamental dans ce sens (Wieman \& Tidow, 1995).

DA : À $15 \mathrm{~km} . \mathrm{h}-1, \mathrm{GM}$ est activé pendant $20 \%$ du cycle de la foulée à plus de $25 \%$ de FVM (Montgomery et al., 1994).

NA : Il est intéressant de noter que le GM est activé au maximum à $40 \%$ de FVM (à 15 $\mathrm{km} . \mathrm{h}-1$ ), mais qu'il manifeste peu de périodes de réelle inactivité, étant sollicité pour cette même vitesse à $10 \%$ de FVM au minimum tout au long du cycle de la foulée (Montgomery et al., 1994).

À $15 \mathrm{~km} . \mathrm{h}-1$, GM est activé à plus de $25 \%$ de FVM pendant $20 \%$ du cycle de la foulée (Montgomery et al., 1994).

MA : Pendant la phase PO3, GM se contracte sur le mode excentrique pour décélérer la cuisse dans son mouvement vers l'avant. Ce rôle se trouve réduit aux vitesses les plus faibles. Au début de l'appui, GM participe à la stabilisation de la cuisse et du bassin.

Le tableau 1, récapitulatif des données précédentes, présente les données de la littérature relatant les niveaux et durées d'activité. Le niveau d'activité est présenté à deux vitesses : $15 \mathrm{~km} . \mathrm{h}-1$ et $21 \mathrm{~km} . \mathrm{h}-1$.

L'examen du tableau 1 nous permet de remarquer trois profils différents :

- les muscles fortement activés (en \% du niveau d'activité enregistré à FVM) tel que Ga notamment ;

- les muscles longuement activés (en \% du cycle de la foulée) tel que TA ;

- les muscles à la fois fortement et longuement sollicités tel que BF.

Il est à noter que les muscles les plus longuement activés (TA et BF) sont également les muscles les plus richement dotés en fibres I.

\section{VARIABILITÉ DU NIVEAU D'ACTIVITÉ MUSCULAIRE}

L'examen de la littérature indique que les valeurs qui témoignent du niveau d'activité musculaire varient selon le contexte de la tâche effectuée à savoir : la vitesse, la fatigue, la technique de réalisation. 


\subsection{Influence de la vitesse sur les caractéristiques de l'activité musculaire}

Quelques auteurs ont observé l'évolution parallèle et croissante de l'EMG avec l'augmentation de la vitesse de course : Oshikawa et al. (1973), Elliot \& Blanksby (1979), Nilsson et al. (1985), Mann et al. (1986), Montgomery et al. (1994) (Hanon et al., sous presse). II semble que l'activation soit multipliée par un facteur qui varie de 1,1 à 1,7 entre 13 et $21 \mathrm{~km} . \mathrm{h}-1$ selon les muscles (Hanon et al., sous presse). Pour Andersson et al. (1997) qui comparent une gamme de vitesse plus étendue, le niveau d'activité peut être multiplié par 4 ou 5 pour certains fléchisseurs de hanche. Dans le cas de $\mathrm{Ga}$, seule l'intensité de la préactivation serait accrue avec la vitesse (Komi et al., 1987), l'activation restant stable.

II apparaît que la durée du signal est inversement proportionnelle à la vitesse de course. Ainsi, Ga est-il activé $268 \mathrm{mec}$ à $13 \mathrm{~km}$.h-1 et $178 \mathrm{msec}$ à $22 \mathrm{~km}$.h-1 (Hanon et al., sous presse). Ceci est vérifié pour TA et VL.

Toutefois, il apparaît que les muscles biphasiques ne suivent pas cette règle. En effet, pour BF et RF, la durée totale d'activité exprimée en valeur absolue augmentent avec la vitesse ce qui est imputable notamment à l'augmentation de l'activité enregistrée au cours de la phase d'oscillation.

Si l'on considère les données exprimées relativement au cycle de la foulée et étant donné que la durée du cycle de la foulée diminue avec la vitesse (cf. figure 4), les muscles sont actifs pendant un pourcentage plus grand du cycle de la foulée. De ce fait, les muscles ont un temps de repos qui diminue avec la vitesse.

Selon Montgomery et al. (1994), en fonction de la vitesse, les muscles augmentent leur niveau d'activité surtout dans la séquence excentrique de leur mise en jeu, ce que ne confirment pas Méro \& Komi (1986) qui mesurent des niveaux d'activité multipliés par 2 entre la vitesse lente et le sprint à la fois en phase excentrique et en phase concentrique. La tension musculaire est donc produite de façon plus brève et plus intense.

Par ailleurs, les muscles tendent à être actifs plus tôt dans le cycle de la foulée (McClay et al., 1987). D'après les données de Reber et al. (1993), la préactivité du Ga serait plus précoce d'environ $5 \%$ aux vitesses élevées. Pour McClay et al. (1987), l'activité des ischios jambiers intervient plus tôt dans le cycle de la foulée.

Avec l'augmentation de la vitesse, des bouffées d'activité peuvent apparaître au cours du cycle de foulée : la contrainte apportée par l'oscillation du membre inférieur libre augmente dans le freinage. De ce fait, l'activité des ischios jambiers engagés dans ce freinage est plus précoce et plus intense. Ce phénomène correspond à une bouffée supplémentaire qui n'existe pas aux vitesses très basses selon ces auteurs. II en est de même pour VM (Vastus Medialis) et VL qui témoignent d'une bouffée d'activité supplémentaire aux vitesses plus élevées pendant PO2 (Simonsen et al., 1985), VM et VL étant responsables de l'extension du genou. De même Elliott \& Blanksby (1979), Méro \& Komi (1987), Mann \& Sprague (1980) observent une action concentrique de Ga en fin de phase de poussée d'une course de sprint.

Par ailleurs, il apparaît que l'augmentation de la vitesse joue également un rôle sur la répartition de l'activité musculaire au cours du cycle : l'augmentation de la vitesse par exemple modifie l'activité de RF qui, pendant l'oscillation du pied, devient supérieure à l'activité observée pendant l'appui. On parle alors pour RF de déplacement de l'activité du RF de l'extension du genou vers la flexion de la hanche (Nilsson et al., 1985 ; Hanon et al., sous presse). De même pour BF, il semble que l'activité la plus forte soit 
enregistrée pendant la phase d'appui aux vitesses basses mais pendant la phase d'oscillation aux vitesses plus élevées.

Par contre, le pattern d'activation de certains muscles comme TA, subissent peu l'influence de la vitesse (Komi et al., 1987).

II est possible de conclure que l'augmentation de la vitesse aboutit :

- à une augmentation plus ou moins prononcée du niveau d'activité musculaire ;

- à une augmentation de la durée d'activité exprimée en \% de la durée totale du

cycle de la foulée et donc à une diminution du temps de relâchement ;

- à une intervention musculaire plus précoce dans le cycle de la foulée ;

- à l'apparition d'une bouffée supplémentaire au cours du cycle de la foulée pour certains muscles (VM, VL et Ga).

\subsection{Influence de la fatigue sur les niveaux d'activité musculaire}

La fatigue est un concept général qui se traduit par une altération sévère de la performance et qui inclut une augmentation de la sensation de l'effort nécessaire pour exercer cette force ou une éventuelle inaptitude à générer ce niveau de force (Enoka \& Stuart, 1992). À mesure que l'activité musculaire est maintenue, on observe une diminution progressive des possibilités de produire de la tension musculaire qui n'est pas sans conséquence sur la foulée. Une augmentation du temps de contact et une diminution de l'amplitude de la foulée de 5 à $8 \%$ sont notées sur piste sur $400 \mathrm{~m}$ (Nummela et al., 1992 ; Sprague \& Man, 1983), sur 800m (Gajer et al., 1996) comme sur 10000 m (Elliot \& Ackland, 1981). Sur tapis roulant, cette chute d'amplitude est également observée (Hanon et al., sous presse ; Hausswirth et al., 2000 ; Kyrölainen et al., 2000), même si ces données sont contredites par d'autres études (Williams et al., 1991 ; Candau et al., 1998) qui concluent à l'augmentation de l'amplitude avec la fatigue. II est à noter que sur les distances inférieures, ce n'est pas l'amplitude qui est réduite sur la fin de course, mais la fréquence des foulées (Bruggeman et al., 1999 entre autres auteurs).

Selon Chapman \& Fraser (1982), les modifications du pattern de la foulée dans la fatigue ne sont pas généralisables et seuls des profils individuels peuvent être déterminés.

Néanmoins, la durée de la contraction musculaire augmente avec l'apparition de la fatigue, ce qui se traduit en course par une augmentation du temps de contact (Chapman \& Fraser, 1982 ; Sprague \& Mann, 1983 ; Nummela et al., 1992 ; Gager et al., 1996).

La durée prolongée de la bouffée musculaire aboutit principalement à une augmentation du rapport temps de travail/temps de relaxation (Bigland-Ritchie et al., 1983). De la même manière, le rapport EMGi/force, fréquemment utilisé pour décrire la fatigue en condition isométrique (Bigland-Ritchie, 1981) et en condition dynamique (Gollhofer et al., 1987), augmente au cours de l'effort de course, indiquant une diminution de l'efficacité de l'activité myoélectrique. Il faut noter aussi l'augmentation du rapport EMGi/ force au cours de la deuxième partie du 400 mètres analysé par Nummela et al., 1992. Horita \& Ishiko (1987) interprètent cette baisse du rendement neuromusculaire comme un témoin de l'apparition de la fatigue périphérique.

Selon Nummela et al. (1992), les niveaux d'activité musculaire (Ga, VL) au cours d'un $400 \mathrm{~m}$ alors que la vitesse diminue, s'élèvent dans toutes les phases du cycle de la foulée. Cette augmentation vaut pour le pic d'activité musculaire (MAXA) déterminé sur une séquence de $50 \mathrm{~ms}$, alors qu'elle n'est pas sensible pour l'activité minimale (MINA) 
toujours repérée pour une séquence de 50 ms. Ces résultats sont confirmés par ceux de Méro \& Peltola (1989). Cette augmentation du niveau d'activité musculaire est particulièrement notable dans les 50 premières ms de la phase de contact. Le facteur de relaxation défini comme la durée du cycle de la foulée pendant laquelle le niveau d'activité musculaire est inférieur à $10 \%$ de MAXA diminue aussi bien pour GA que pour VL de 67 à $58 \%$ du cycle de la foulée.

Par ailleurs, selon Sprague \& Mann (1983), dans la fatigue, l'athlète perd la capacité à produire les niveaux de force adéquats.

Les principales conséquences de la fatigue observées au niveau des activités musculaires résident dans une augmentation du rapport temps de travail/temps de relaxation, due à la diminution du temps de relaxation musculaire et à une augmentation du rapport EMGi / force qui indique une diminution de l'efficacité de l'activité myoélectrique.

\subsection{Influence de l'expertise sur le niveau d'activité musculaire}

Les études qui traitent du niveau d'activité musculaire et de l'expertise du coureur sont assez peu nombreuses. Elles permettent néanmoins de poser l'hypothèse d'une relation entre l'expertise du sujet dans l'activité évaluée et le niveau d'activité musculaire. II semble, en effet, selon Hoshikawa et al. (1973), qu'à vitesse donnée, le niveau d'activité musculaire dépende du niveau d'expertise, les sujets les plus experts se caractérisant par un moindre niveau "général " d'activité musculaire. Une durée d'activité significativement supérieure pendant la phase d'oscillation et plus faible pendant l'appui a pu être observée chez des coureurs experts comparés à des sportifs non coureurs (données personnelles non publiées). Ces résultats semblent confirmer le fait mis en évidence par Paavolainen et al. (1999), qu'en phase de propulsion, VL et Ga semblent moins activés chez des coureurs de 10000 mètres experts que chez des coureurs de moindre niveau.. De la même manière, selon cette même étude, la préactivité de $\mathrm{Ga}$ est apparu plus longue chez les experts. Ces deux résultats pouvant être expliqués par une action de « griffé " préalable à la phase d'appui chez les coureurs entraînés à la course.

Dans un autre domaine, Heise et al. (1995) ont centré leurs investigations sur les relations entre le coût énergétique de l'athlète et le niveau d'activité musculaire de certains muscles. En course, cette notion de coût énergétique exprime le rapport entre la dépense énergétique (en mlO2.mn-1.kg-1) et la vitesse de déplacement (en m.s-1 ou en km.h-1). Plus ce rapport est faible, plus le sujet est économique. Heise et al. (1995) mentionnent que les durées de coactivité entre les muscles bi-articulaires pendant les phases d'appui et d'oscillation ont une influence sur le coût énergétique. Kyrölaïnen et al. (2001), observent également une augmentation de coactivité entre agoniste/antagoniste (VL et BF d'une part, $\mathrm{Ga}$ et TA de l'autre) qui aboutirait à l'augmentation de la raideur du genou et de la cheville au début de la phase de contact. Selon ces différents auteurs, cette activité synchrone des muscles bi-articulaires pourrait aboutir à la restitution d'une plus grande quantité d'énergie au cours de la phase de propulsion et ainsi à l'épargne de l'énergie métabolique.

Par ailleurs, il a été démontré par Sprague \& Mann (1983) que le pattern de course se dégradait dans de moindre proportion dans la fatigue chez les coureurs experts comparés aux coureurs novices. Chez ces derniers, avec la fatigue, il est noté :

- une moindre flexion de la hanche avant l'appui ;

- une augmentation de l'extension du genou avant l'appui ; 
- une moindre extension de hanche pendant l'appui ;

- une flexion plantaire de la cheville accrue en fin d'appui ;

- une extension de la hanche accrue en fin d'appui.

Néanmoins, ces résultats ne sont pas étayés par des enregistrements électromyographiques.

Le niveau d'expertise semble influencer les activités musculaires enregistrées au cours de la course.

Les principales modifications concernent notamment les patterns d'activité des muscles bi-articulaires ainsi que le rapport pré-activité/activité des muscles mono-articulaires. Néanmoins, ces résultats sont très parcellaires et mériteraient une étude plus approfondie.

\section{CONCLUSION}

Concernant les patterns, les niveaux et les durées d'activité des muscles des membres inférieurs enregistrées lors de la course, il semble que la littérature soit relativement consensuelle. Les quelques variations observées sont inévitablement liées aux vitesses et conditions de course (piste ou tapis roulant) et au niveau d'expertise des sujets.

Par contre, les données relatives aux modalités de contractions musculaires, que ne permet pas d'approcher la seule analyse électromyographique, apparaissent plus contradictoires et plus discutables. La difficulté matérielle liée à la précision de l'analyse rend la détermination de ces modes d'activité peu accessible. En effet, s'il est nécessaire de déterminer avec précision les mouvements articulaires par analyse cinématographique, il est ensuite indispensable de maîtriser un modèle anatomique qui permet de proposer l'hypothèse d'un mode d'activité concentrique, isométrique ou excentrique pour un muscle donné au cours d'une mobilisation articulaire.

Sur un plan plus pratique, l'analyse électromyographique objectivant la détermination des chefs musculaires les plus précocement fatigués pour une vitesse donnée devrait permettre une meilleure adaptation des contenus d'entraînement, notamment des programmes de renforcement musculaire.

Merci à Christian Miller (responsable du laboratoire de biomécanique et de physiologie de I'INSEP) et à Jacques Quièvre (enseignant-chercheur, laboratoire de biomécanique et physiologie) pour leurs conseils avisés.

\section{BIBLIOGRAPHIE}

AE M., ITO A., SUZUKI M. (1992). The men's 100 metres. IAAF, 7, 47-52.

ANDERSSON E.A., NILSSON J., THORSTENSSON A. (1997). Intramuscular EMG from the hip flexor muscles during human locomotion. Acta Physiol Scand, 161, 361-370.

BIGLAND-RITCHIE B. (1981). EMG/force relations and fatigue of human volontary contactions. Exer Sports Sci Rev, 9, 75-117.

BOBER T., MULAREZ Y.K.W. (1990). The mechanics of the leg swing in running. Techniques in Athletics, Cologne, 7-9 june. Conference proceedings, 2, 507-510.

BOUISSET S., MATON B. (1995). Muscle, posture et mouvement. Base et applications de la méthode électromyographique. Paris, Herman. 
BRUGGEMANN G.P., KOSZEEWSKI D., MULLER H. (1999). Biomechanical Research Project Athens 1997. Final Report. Oxford, Meyer \& Meyer Sport (UK) Ltd.

CANDAU R., BELLI A., MILLET G.Y., GEORGES D., BARBIER B., ROUILLON J.D. (1998). Energy cost and running mechanics during a treadmill run to voluntary exhaustion in humans. Eur J Appl Physiol, 77, 479-485.

CHAPMAN A.E. \& FRASER R. (1982). Hierarchy of changes induced by fatigue in sprinting. Can J Appl Sci, 7, 116-122.

ELLIOT B.C., ACKLAND T. (1981). Biomechanical effects of fatigue on 10,000 meter running technique. Res Quart Ex Sport, 52, 160-166.

ELLIOT B.C., BLANKSBY B.A. (1979). The synchronisation of mucle activity and body segment movement during running cycle. Med Sci Sports, 11, 322-327.

ENOKA R.M., STUART D.G. (1992). Neurobiology of muscle fatigue. J Appl Physiol, 72, 1631-1648.

GAJER B., DUREY A., HANON C., THEPAUT-MATHIEU C. (1996). Stride evolution in an $800 \mathrm{~m}$ race. Physical activity, sport and health. The 1996 international preolympic congress. 10-14 juillet. Dallas Texas.

GOLLHOFER A., KOMI P.V., MIYASHITA M., AURA O. (1987). Fatigue during strechshortening cycle exercises : changes in mechanical performance of human skeletal muscle. Int J Sports Med, 8, 71-78.

HANON C., THEPAUT-MATHIEU C., VANDEWALLE H. (sous presse). Determination of muscular fatigue in running. Eur $J$ Appl Physiol.

HAUSSWIRTH C., BRISSWALTER J., VALLIER J.M., SMITH D., LEPERS R. (2000). Evolution of electromyographic signal, running economy, and perceived exertion during different prolonged exercises. Int J Sports Med, 21, 429-436.

HEISE G., MORGAN D.W., HOUGH H., CRAIB M. (1995). Relationship between running economy and temporal EMG characteristics of bi-articular leg muscles. Int J Sports Med, 17, 128-133.

HORITA T., ISHIKO T. (1987). Relationships between muscle lactate accumulation and surface EMG activities during isokinetic contractions in man. Eur J Appl Physiol, 56, 18-23.

HOSHIKAWA T., MATSUI H., MIYASHITA M. (1973). Analysis of running pattern in relation to speed. Med Sport 8 : Biomechanics, III, 342-348 (Karger, Basel).

JOHNSON M.A., POLGAR J., WEIGHTMAN D., APPLETON D. (1973). Data on the distribution of fibres types in thirty-six human muscles. An autopsy study. $J$ Neurol. Sci, 18, 111-129.

KAPANDJI I.A. (1965) Physiologie articulaire. Schémas commentés de mécanique humaine. Fascicule II (Membre inférieur). Paris, Librairie Maloine S.A 27, rue de l'école de médecine.

KYRÖLÄINEN H., PULLINEN T., CANDAU R., AVELA J., HUTTUNEN P., KOMI P.V. (2000). Effects of marathon running economy and kinematics. Eur J Appl Physiol, 4, 297-304.

KYRÖLÄINEN H., BELLI A., KOMI P.V. (2001). Biomechanical factors affecting running. Med Sci Sports Exerc, 8, 1330-1337.

KOMI P.V., GOLLHOFER A., SCMIDTBLEICHER D., FRICK V. (1987). Interaction between man and schoe running : considerations for a more comprehensive measurement approach. Int J Sports Med, 8, 196-202.

LEMAIRE E.D., ROBERTSON D.G.E. (1987). Power in sprinting. TFS, 35, 13-17.

MCCLAY I., LAKE M., CAVAGNAH P. (1990). Muscle activity in running. Chapitre 6 Biomechanics, VA, 165-186.

MANN R.A., HAGY J. (1980). Biomechanics of walking, running, and sprinting. Am J Sports Med, 8, 345-350. 
MANN R.A., SRAGUE P. (1980). A kinetic analysis of the ground leg during sprint running. Res Quart Exerc Sport, 51, 334-348.

MANN R.A., MORAN G., DOUGHERTY S. (1986). Comparative electromyography of the lower extremity in jogging, running, and sprinting. Am J Sports Med, 14, 501510.

MERO A., KOMI P.V. (1986). Force -, EMG -, and elasticity-velocity relationships at submaximal, maximal and supramaximal running speeds in sprinters. Eur J Appl Physiol, 55, 553-561.

MERO A., KOMI P.V. (1987). Electromyographic activity in sprinting at speeds ranging from sub-maximal to supra-maximal. Med Sci Sports Exerc, 19, 266-274.

MERO A., PELTOLA E. (1989). Neural activité fatigued and non-fatigued conditions of short and long sprint running. Biol Sport, 6, 43-53.

MONTGOMERY W.H., PINK M., PERRY J. (1994). Electromyographic analysis of hip and knee musculature during running. Am J Sports Med, 22, 272-278.

NILSSON J., THORSTENSSON A., HALBERTSMA J. (1985). Changes in leg movements and muscle activity with speed of locomotion and mode of progression in humans. Acta Physiol Scand, 123, 457-475.

NUMMELA A., VUORIMAA T., RUSKO H. (1992). Changes in force production, blood lactate and EMG activity in the 400-m sprint. J Sports Sci, 10, 217-228.

PAAVOLAINEN L., NUMMELA A., RUSKO H., HAKKINEN K. (1999). Neuromuscular characteristics and fatigue during $10 \mathrm{~km}$ running. Int J Sports Med, 20, 516-521.

REBER L., PERRY J., PINK M. (1993). Muscular control of the ankle in running. Am J Sports Med, 21, 805-810.

SIMONSEN E., THOMSEN L., KLAUSEN J. (1985). Activity of mono-and biarticular leg muscles during sprint running. Eur J Appl Physiol, 54, 524-532.

SPRAGUE P., MANN R. (1983). The effects of muscular fatigue on the kinetics of sprint running. Res Quart Exerc Sport, 54, 60-66.

STEINDLER A. (1964). Kinesiology of the human body. Charles C Thomas Edit Springfield, 334-345.

WANK V., FRICK V., SCHMIDTBLEICHER D. (1998). Kinematics and EMG of lower limb muscles in overground and treadmill running Int $J$ Sports Med, 19, 455-461.

WASER J. (1985). Zum Techniktraining beim laufen. Leistungssport, 1, 34-38.

WIEMANN K., TIDOW G. (1995). Relative activity of hip and knee extensors in sprinting-implications for training. IAAF, 10, 29-49.

WILLIAMS K.R., SNOW R., AGRUSS C. (1991). Changes in distance running kinematics with fatigue. Int J Sport Biomechanics, 7, 138-162. 


\section{Figures et tableaux :}

\section{Figure 1. Description des différentes phases de la foulée}

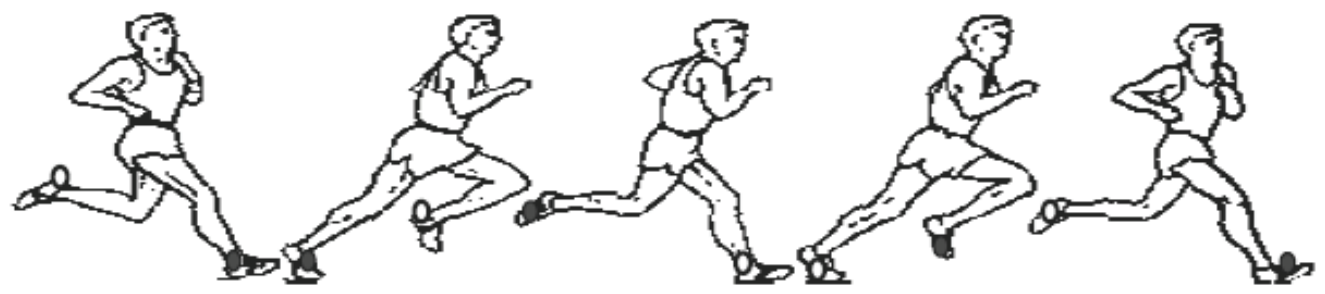

\begin{tabular}{|l|l|l|l|}
\hline Phase d'appui(PA) & \multicolumn{3}{|c|}{ Phase d'oscillation (PO) } \\
\hline & $\begin{array}{l}\text { Première phase } \\
\text { d'oscillation (PO1) }\end{array}$ & $\begin{array}{l}\text { Deuxième phase } \\
\text { d'oscillation (PO2) }\end{array}$ & $\begin{array}{l}\text { Troisième phase } \\
\text { d'oscillation (PO3) }\end{array}$ \\
\hline $\begin{array}{l}\text { Elle commence à la pose } \\
\text { du talon ipsi-latéral (noir) } \\
\text { et se termine lorsque les } \\
\text { orteils du même pied } \\
\text { quittent le sol }\end{array}$ & $\begin{array}{l}\text { Elle commence lorsque } \\
\text { les orteils du pied } \\
\text { ipsilatéral (noir) quittent } \\
\text { le sol jusqu'à la pose du } \\
\text { talon contralatéral } \\
\text { (blanc).PO1est une } \\
\text { phase aérienne (pied } \\
\text { derrière) }\end{array}$ & $\begin{array}{l}\text { Elle commence à la } \\
\text { pose du talon } \\
\text { contralatéral (blanc) } \\
\text { jusqu'à ce que les } \\
\text { orteils du même pied } \\
\text { contralatéralquittent } \\
\text { le sol : PO2 est une } \\
\text { phase d'appui }\end{array}$ & $\begin{array}{l}\text { Elle commence lorsque } \\
\text { lesteils du pied } \\
\text { contralatéral (blanc) } \\
\text { quittent le sol et se } \\
\text { termine à la pose du } \\
\text { talon du pied ipsilatéral } \\
\text { (noir): PO3 est une } \\
\text { phase aérienne (pied } \\
\text { devant) }\end{array}$ \\
\hline
\end{tabular}

- Appui contralatéral

Appui ipsi latéral 
Figure 2. Enregistrement électromyographique des activités musculaires en course sur tapis roulant

Ces enregistrements (Hanon et al., soumis à publication) correspondent à 3 phases d'appui et 2 phases d'oscillation complètes à $15 \mathrm{~km}$.h-1 déterminées par le signal on/off de deux contacteurs placés sous le gros orteil et sous le talon droit des sujets. Le signal « on » signifie qu'il y a contact au sol.

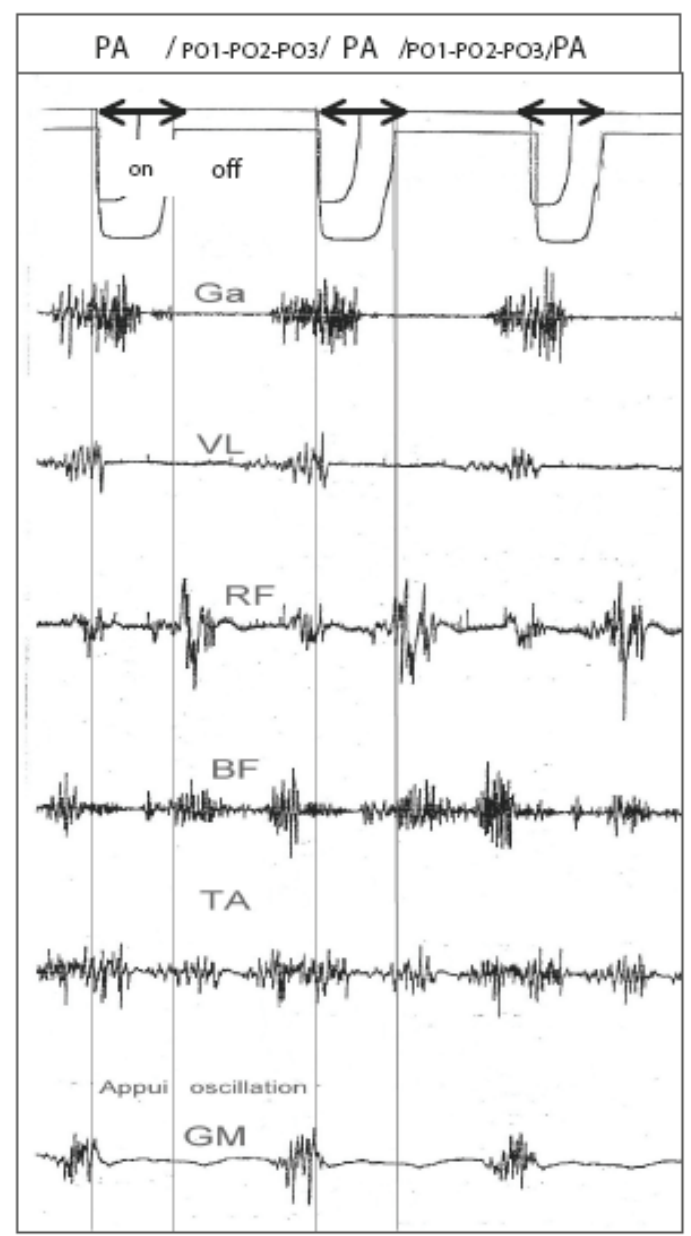

$\mathrm{Ga}=$ Gactrocnemius, VL = Vastus Lateralis, RF = Rectus Femoris, BF = Biceps Femoris, TA = Tibialis anterior, GM = Gluteus 
Figure 3. Activité musculaire de Ga, TA, VL, RF, BF au cours d'un cycle de foulée réalisée à vitesse moyenne $(15 \mathrm{~km} \cdot \mathrm{h}-1)$

Les zones grisées représentent une activité plus ou moins importante selon la couleur, gris clair $=$ activité comprise entre 10 et $40 \%$ du niveau d'activation maximale recueilli en course pour le muscle considéré ( $\%$ FVM), gris foncé = activité comprise entre 40 et $80 \%$ FVM, noir = activité comprise entre 80 et $100 \%$ FVM).

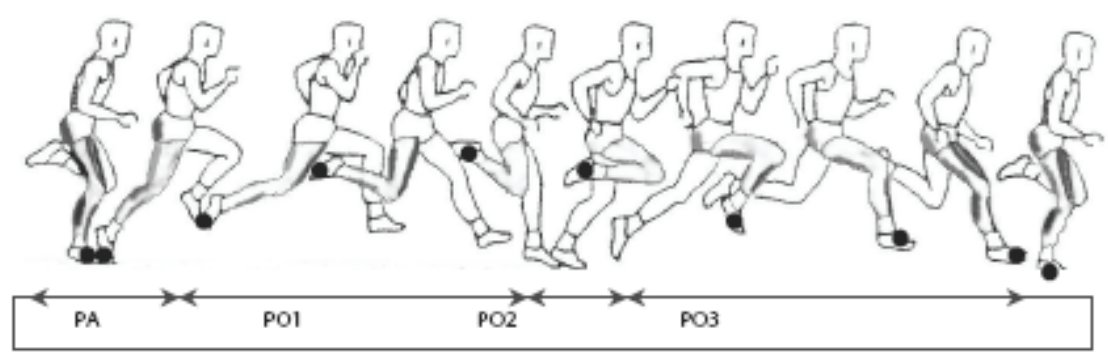

Figure 4. Durée et niveau d'activité des muscles étudiés

Les données représentées sont issues de résultats obtenus à $15 \mathrm{~km} . \mathrm{h}-1$. Les périodes d'activation correspondent à la détection d'un signal électromyographique pris en compte lorsqu'il est supérieur ou égal à $10 \%$ du pic de l'enveloppe de la bouffée considérée.

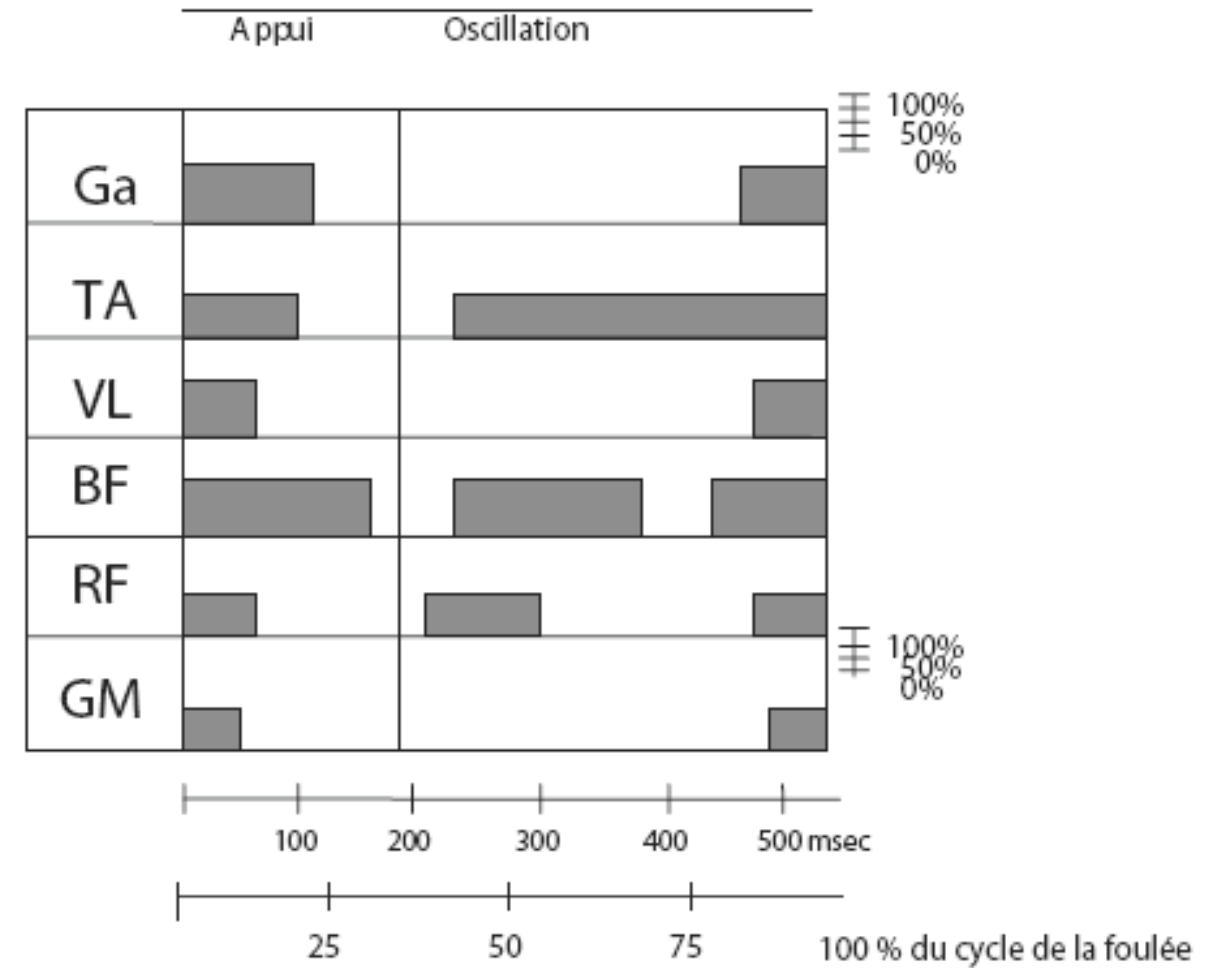




\section{Tableau 1. Évolution des niveaux et durées d'activité avec la vitesse}

FVM = Force Volontaire Maximale : niveau d'activation maximal recueilli en mode isométrique.

Tous résultats : Hanon et al. (sous presse), à l'exception de GM (Montgomery et al., 1994) pour des enregistrements à $15 \mathrm{~km} . \mathrm{h}-1$.

\begin{tabular}{|c|c|c|c|c|}
\hline \multirow[b]{2}{*}{ Muscle } & \multicolumn{2}{|c|}{$\begin{array}{l}\text { Niveau d'activité (NA) } \\
\text { (en \% de FVM) }\end{array}$} & \multicolumn{2}{|c|}{$\begin{array}{c}\text { Durée d'activité (DA) } \\
\text { (en \% de la durée totale du cycle) }\end{array}$} \\
\hline & $13 \mathrm{~km} \cdot \mathrm{h}^{-1}$ & $21 \mathrm{~km} \cdot \mathrm{h}^{-1}$ & $13 \mathrm{~km} \cdot \mathrm{h}^{-1}$ & $21 \mathrm{~km} \cdot \mathrm{h}^{-1}$ \\
\hline $\mathrm{BF}$ & 44 & 62 & 66 & 75 \\
\hline Ga & 46 & 56 & 42 & 38 \\
\hline $\mathrm{RF}$ & 21 & 36 & 40 & 56 \\
\hline VL & 40 & 60 & 33 & 30 \\
\hline $\mathrm{TA}$ & 37 & 42 & 85 & 90 \\
\hline GM & $20\left(15 \mathrm{~km}^{-1} \mathrm{~h}^{-1}\right)$ & - & $25\left(15 \mathrm{~km}^{-1}\right)$ & - \\
\hline
\end{tabular}

